\title{
Comparison of yield-vertex tangential loading and principal stress rotational loading
}

\author{
Nan Lu ${ }^{\mathrm{a}}$, Yunming Yang ${ }^{\mathrm{b}, *}$, Hai-Sui Yu ${ }^{\mathrm{c}}$ \\ ${ }^{a}$ Department of Civil Engineering, International Doctoral Innovation Centre, University of \\ Nottingham Ningbo China, Ningbo 315100, PR China \\ ${ }^{\mathrm{b}}$ Ningbo Nottingham New Materials Institute, University of Nottingham Ningbo China, Ningbo 315100, \\ PR China \\ ${ }^{c}$ School of Civil Engineering, University of Leeds, Leeds LS2 9JT, UK \\ *Corresponding to: ming.yang@nottingham.edu.cn (Yunming Yang)
}

\begin{abstract}
The yield-vertex tangential loading theory is a constitutive approach that accounts for the plastic straining induced by the part of a stress rate directed tangential to the yield surface. One of the important applications of this theory is in the study of geotechnical problems involving significant rotation of principal stress directions. However, it is inaccurate to simply regard the tangential loading as an equivalence to the principal stress rotation. For future reference, this paper presents an investigation into the difference between the tangential loading theory and a true purely principal stress rotational loading theory. Mathematical derivation shows that the tangential stress rate includes the rotational stress rate and an additional coaxial term that is associated with the variation of the Lode angle. Numerical applications of these two theories indicate that in shear dominated problems, such as simple shear, the two theories are almost identical and interchangeable, but in non-shear dominated circumstances, such as footing, the tangential loading theory produces considerably softer results than the rotational loading theory.
\end{abstract}

Keywords: Plasticity; Tangential loading; Principal stress rotation; Simple shear; Footing.

\section{Introduction}

The yield-vertex tangential loading (TL) theory, firstly introduced by Rudnicki and Rice (1975), is a complementary framework to the conventional plasticity theory. It assumes that, in addition to the plastic loading along the 'straight ahead' stressing, the part of a stress rate directed tangential to the yield surface also induces plastic straining. This theory has strong influences on the predicted stressstrain responses of soil and is found to be of importance in some geotechnical applications such as bifurcation (Yatomi et al., 1989, Papamichos and Vardoulakis, 1995). Extensive investigations, implementations, and some pertinent improvements regarding this theory can be found in the literature (Hashiguchi and Tsutsumi, 2001, Yang and Yu, 2006, Qian et al., 2008). In particular, it has been successfully applied to consider the responses of soil subjected to principal stress rotational loading (RL) (e.g. Tsutsumi and Hashiguchi (2005)), which refers to the condition of continuously rotated principal stress axes but fixed principal stress magnitudes.

However, the TL theory is not designated solely for the study of RL. It can also be triggered by a circular stress path in the $\pi$-plane with centre at the origin, which is characterised by a continuous change of principal stress magnitudes but fixed principal stress directions. Given that the rotation of principal stress directions is of great academic and practical importance, experimental and numerical studies where principal stress axes rotated with other parameters remaining constant have drawn serious attention in the last three decades (Miura et al., 1986, Tong et al., 2010, Qian et al., 2017, Li and Dafalias, 2004, Tian and Yao, 2018, Li and Yu, 2010). In the literature, there exists a technique of 
isolating the part of a stress rate that is responsible for the rotation of principal stress directions (see for example Yu and Yuan (2006), Yang and Yu (2013) and Yuan et al. (2018)). Computation shows that the inclusion of the plastic loading associated with this rotational stress rate significantly improves the accuracy of numerical prediction (Yang and Yu, 2013).

Considering that the TL theory has been frequently used in geotechnical applications involving significant principal stress rotation, its difference from the true purely principal stress RL should be investigated. Thus, this paper presents a comparative analysis between the TL and RL theories. Their mathematical differences are discussed, and their performances in the simple shear and strip footing problems are compared.

\section{Tangential loading and rotational loading}

Within the elastoplasticity framework, the total strain rate is decomposed into

$$
\dot{\varepsilon}_{i j}=\dot{\varepsilon}_{i j}^{e}+\dot{\varepsilon}_{i j}^{p}
$$

where the superscripts $e$ and $p$ denote elastic and plastic components, respectively. The elastic relation is assumed to be isotropic, which gives

where

$$
\dot{\sigma}_{i j}=E_{i j k l} \dot{\varepsilon}_{k l}^{e}
$$

$$
E_{i j k l}=K \delta_{i j} \delta_{k l}+G\left(\delta_{i k} \delta_{j l}+\delta_{i l} \delta_{j k}-\frac{2}{3} \delta_{i j} \delta_{k l}\right)
$$

with $\delta_{i j}$ being the Kronecker delta, and $K$ and $G$ the elastic bulk and shear moduli, respectively. To facilitate later presentation, the plastic strain rate $\dot{\varepsilon}_{i j}^{p}$ is further decomposed into two components. The first component, denoted by $\dot{\varepsilon}_{i j}^{p c}$, is associated with the conventional plasticity theory (superscript $c$ ), characterized with a modified Drucker-Prager yield surface given as

$$
f=\sqrt{(a \sin \phi)^{2}+\tau^{2}}-p \sin \phi-c \cos \phi
$$

where $\tau=\sqrt{\left(s_{i j} s_{i j}\right) / 2}$ and $s_{i j}$ is the deviatoric stress tensor; $p$ is the mean normal stress; $c$ is the soil cohesion and $\phi$ is the friction angle. Both $c$ and $\phi$ are constant in this work, utilizing a perfect plasticity framework. The term $(a \sin \phi)^{2}$ inside the radical sign represents a hyperbolic approximation in the meridional (constant Lode angle) plane. When $a \leq 0.5 c \cdot \cot \phi, \mathrm{Eq}(4)$ closely represents the original Drucker-Prager yield surface. The plastic potential is then obtained by replacing the friction angle $\phi$ with a dilatation angle $\psi$ in $\mathrm{Eq}(4)$.

The second component of $\dot{\varepsilon}_{i j}^{p}$, is either $\dot{\varepsilon}_{i j}^{p t}$ when the TL mechanism is used, or $\dot{\varepsilon}_{i j}^{p r}$ when the RL mechanism is used. The meanings of superscripts $t$ and $r$ are self-evident. The development of $\dot{\varepsilon}_{i j}^{p t}$ and $\dot{\varepsilon}_{i j}^{p r}$ is addressed in the following.

\subsection{TL model}

Rudnicki and Rice (1975) proposed that the tangential plastic strain rate $\dot{\varepsilon}_{i j}^{p t}$ is given by

$$
\dot{\varepsilon}_{i j}^{p t}=\frac{1}{h} \dot{s}_{i j}^{t}
$$

where $h$ is a plastic modulus governing the additional loading mechanism; and $\dot{s}_{i j}^{t}$ is the part of $\dot{s}_{i j}$ directed tangential to the yield surface, defined as

$$
\dot{s}_{i j}^{t}=\dot{s}_{i j}-\frac{s_{i j} s_{k l}}{2 \tau^{2}} \dot{s}_{k l}=N_{i j k l}^{t} \dot{\sigma}_{k l}
$$

where

$$
N_{i j k l}^{t}=\frac{1}{2}\left(\delta_{i k} \delta_{j l}+\delta_{i l} \delta_{j k}-\frac{2}{3} \delta_{i j} \delta_{k l}-\frac{s_{i j} s_{k l}}{\tau^{2}}\right)
$$


Substituting $\mathrm{Eq}(6)$ into $\operatorname{Eq}(5)$, one has

$$
\dot{\varepsilon}_{i j}^{p t}=\frac{1}{h} N_{i j k l}^{t} \dot{\sigma}_{k l}
$$

The fourth-order tensor $N_{i j k l}^{t}$ is known as the deviatoric tangential projection as it projects an arbitrary second-order tensor into its deviatoric part tangential to the yield surface (Hashiguchi, 2014). In Fig 1(a), a schematic illustration of the tangential plastic strain rate is shown. It should be emphasized that the principal (deviatoric) stress presentation in Fig 1(a) illustrates only part of the TL mechanism since Eqs(5 8) are defined in the general stress space.

Using $\mathrm{Eq}(8)$, the overall elastoplastic stress rate-strain rate relation becomes

$$
\dot{\sigma}_{i j}=\left[E_{i j k l}-\frac{\left(E_{i j a b} R_{a b}\right)\left(l_{c d} E_{c d k l}\right)}{\left(K_{p}+l_{m n} E_{m n s t} R_{s t}\right)}-\frac{4 G^{2}}{h+2 G} N_{i j k l}^{t}\right] \dot{\varepsilon}_{k l}
$$

where $l_{i j}$ and $R_{i j}$ are the normals to the yield and plastic potential surfaces, respectively; $K_{p}$ is the plastic modulus governing the conventional plastic loading and is zero in this paper as a result of the perfect plasticity setting.

\subsection{RL model}

For the purpose of comparison, the rotational plastic strain rate $\dot{\varepsilon}_{i j}^{p r}$ is given similar to $\mathrm{Eq}(5)$, written as

$$
\dot{\varepsilon}_{i j}^{p r}=\frac{1}{h} \dot{\sigma}_{i j}^{r}
$$

where $h$ is the same parameter as in $\operatorname{Eq}(5) ; \dot{\sigma}_{i j}^{r}$ is the part of $\dot{\sigma}_{i j}$ that triggers the RL at fixed principal stress magnitudes. This rotational rate $\dot{\sigma}_{i j}^{r}$ is taken as purely deviatoric since the RL occurs at constant mean normal stress $p$. It is firstly assumed that the direction of $\sigma_{z}$ is fixed such that the RL takes place in the plane of $\left(\frac{\sigma_{x}-\sigma_{y}}{2}, \tau_{x y}\right)$ shown in Fig $1(\mathrm{~b})$. Then, the $\dot{\sigma}_{i j}^{r}$ must satisfy the following condition

$$
\left[\begin{array}{c}
\left(\dot{\sigma}_{x}^{r}-\dot{\sigma}_{y}^{r}\right) / 2 \\
\dot{\tau}_{x y}^{r}
\end{array}\right]=\left[\begin{array}{c}
\left(\dot{\sigma}_{x}-\dot{\sigma}_{y}\right) / 2 \\
\dot{\tau}_{x y}
\end{array}\right]-\frac{\frac{1}{4}\left(\sigma_{x}-\sigma_{y}\right)\left(\dot{\sigma}_{x}-\dot{\sigma}_{y}\right)+\tau_{x y} \dot{\tau}_{x y}}{\frac{1}{4}\left(\sigma_{x}-\sigma_{y}\right)^{2}+\tau_{x y}^{2}}\left[\begin{array}{c}
\left(\sigma_{x}-\sigma_{y}\right) / 2 \\
\tau_{x y}
\end{array}\right]
$$

Using $\mathrm{Eq}(11)$ and noting that $\dot{\sigma}_{x}^{r}+\dot{\sigma}_{y}^{r}=0$, the following expression for the components of $\dot{\sigma}_{i j}^{r}$ is obtained

$$
\left[\begin{array}{c}
\dot{\sigma}_{x}^{r} \\
\dot{\sigma}_{y}^{r} \\
\dot{\tau}_{x y}^{r}
\end{array}\right]=\left[\begin{array}{ccc}
\frac{1}{2} \sin ^{2} 2 \alpha_{\sigma} & -\frac{1}{2} \sin ^{2} 2 \alpha_{\sigma} & -\cos 2 \alpha_{\sigma} \sin 2 \alpha_{\sigma} \\
-\frac{1}{2} \sin ^{2} 2 \alpha_{\sigma} & \frac{1}{2} \sin ^{2} 2 \alpha_{\sigma} & \cos 2 \alpha_{\sigma} \sin 2 \alpha_{\sigma} \\
-\frac{1}{2} \cos 2 \alpha_{\sigma} \sin 2 \alpha_{\sigma} & \frac{1}{2} \cos 2 \alpha_{\sigma} \sin 2 \alpha_{\sigma} & \cos ^{2} 2 \alpha_{\sigma}
\end{array}\right]\left[\begin{array}{c}
\dot{\sigma}_{x} \\
\dot{\sigma}_{y} \\
\dot{\tau}_{x y}
\end{array}\right]
$$

where $\alpha_{\sigma}$ is the direction of major principal stress with reference to the $y$-axis. Geometrical relation in Fig 1(b) indicates that $\sin 2 \alpha_{\sigma}=\frac{\tau_{x y}}{\sqrt{\left(\sigma_{x}-\sigma_{y}\right)^{2} / 4+\tau_{x y}^{2}}}$ and $\cos 2 \alpha_{\sigma}=\frac{\left(\sigma_{x}-\sigma_{y}\right) / 2}{\sqrt{\left(\sigma_{x}-\sigma_{y}\right)^{2} / 4+\tau_{x y}^{2}}}$. In the same way, the rotational stress rates in the planes of $\left(\frac{\sigma_{y}-\sigma_{z}}{2}, \tau_{y z}\right)$ and of $\left(\frac{\sigma_{z}-\sigma_{x}}{2}, \tau_{z x}\right)$ can also be obtained. Combining them altogether, a tensor expression for $\dot{\sigma}_{i j}^{r}$ can be written as

$$
\dot{\sigma}_{i j}^{r}=N_{i j k l}^{r} \dot{\sigma}_{k l}
$$

For detailed expression of the fourth-order tensor $N_{i j k l}^{r}$, readers are referred to Yang and Yu (2013). Compared with the $N_{i j k l}^{t}$ in $\mathrm{Eq}(6)$, it is found that the role of $N_{i j k l}^{r}$ is to project an arbitrary secondorder tensor to its deviatoric part directed along the direction of principal stress rotation. In this sense, it can be called the deviatoric rotational projection. By substituting Eq(13) into Eq(10), one has

$$
\dot{\varepsilon}_{i j}^{p r}=\frac{1}{h} N_{i j k l}^{r} \dot{\sigma}_{k l}
$$

Obviously, $\operatorname{Eq}(14)$ is a counterpart to $\operatorname{Eq}(8)$ when the $\mathrm{RL}$ mechanism is used. Finally, the overall elastoplastic stress rate-strain rate relation in the RL model is given by 


$$
\dot{\sigma}_{i j}=\left[E_{i j k l}-\frac{\left(E_{i j a b} R_{a b}\right)\left(l_{c d} E_{c d k l}\right)}{\left(K_{p}+l_{m n} E_{m n s t} R_{s t}\right)}-\frac{4 G^{2}}{h+2 G} N_{i j k l}^{r}\right] \dot{\varepsilon}_{k l}
$$

Inspection of the foregoing equations indicates that the TL and RL models in this paper are constructed in a very similar way to serve the purpose of comparison. The only difference between the two sets of model formulations is associated with the difference between $N_{i j k l}^{t}$ and $N_{i j k l}^{r}$.

It should be noted that the presentation given here implies circular yield surfaces in the $\pi$-plane and in the plane of $\left(\frac{\sigma_{x}-\sigma_{y}}{2}, \tau_{x y}\right)$ or any other relevant planes. Nevertheless, non-circular shapes can be easily accounted for along the line of Hashiguchi and Tsutsumi (2001).

\subsection{Comparison of $T L$ and $R L$}

$\mathrm{Yu}$ (2006) commented that, under two-dimensional plane strain conditions, the tangential stress rate $\dot{s}_{i j}^{t}$ will downgrade to the rotational stress rate $\dot{\sigma}_{i j}^{r}$ using the definition of $s_{x}=\frac{\sigma_{x}-\sigma_{y}}{2}, s_{y}=\frac{\sigma_{y}-\sigma_{x}}{2}, s_{x y}=$ $\tau_{x y}$ and $\tau=\sqrt{\left(\sigma_{x}-\sigma_{y}\right)^{2} / 4+\tau_{x y}^{2}}$. However, under general three-dimensional conditions, they are not identical.

Noting the deviatoric nature of $\dot{\sigma}_{i j}^{r}$ and $N_{i j k l}^{r}$, Eq(13) can be reformulated as $\dot{s}_{i j}^{r}=\dot{\sigma}_{i j}^{r}=N_{i j k l}^{r} \dot{s}_{k l}+$ $\left.\frac{1}{3} \dot{\sigma}_{m m} \delta_{k l}\right)=N_{i j k l}^{r} \dot{s}_{k l}$. Thus, the deviatoric stress rate $\dot{s}_{i j}$ can be decomposed into

$$
\dot{s}_{i j}=\dot{s}_{i j}^{r}+\left(\mathrm{I}_{i j k l}-N_{i j k l}^{r}\right) \dot{s}_{k l}
$$

where $\mathrm{I}_{i j k l}$ is the fourth-order identity tensor. Substituting $\mathrm{Eq}(16)$ into $\mathrm{Eq}(6)$, and noting that $s_{k l} \dot{s}_{k l}^{r}=0$, the tangential stress rate $\dot{s}_{i j}^{t}$ becomes

$$
\dot{s}_{i j}^{t}=\dot{s}_{i j}^{r}+\left(\mathrm{I}_{i j m n}-N_{i j m n}^{r}\right) \dot{s}_{m n}-\frac{s_{i j} s_{k l}}{2 \tau^{2}}\left(\mathrm{I}_{k l s t}-N_{k l s t}^{r}\right) \dot{s}_{s t}
$$

In the above equation, the terms $\left(\mathrm{I}_{i j m n}-N_{i j m n}^{r}\right) \dot{s}_{m n}-\frac{s_{i j} s_{k l}}{2 \tau^{2}}\left(\mathrm{I}_{k l s t}-N_{k l s t}^{r}\right) \dot{s}_{s t}$ are of the same form as the middle expression of $\mathrm{Eq}(6)$ with the substitution of $\left(\mathrm{I}_{i j m n}-N_{i j m n}^{r}\right) \dot{s}_{m n}$ for $\dot{s}_{i j}$. Therefore, Eq(17) can be further written as

$$
\dot{s}_{i j}^{t}=\dot{s}_{i j}^{r}+N_{i j m n}^{t}\left(\mathrm{I}_{m n k l}-N_{m n k l}^{r}\right) \dot{s}_{k l}
$$

This expression indicates that the tangential stress rate $\dot{s}_{i j}^{t}$ includes the rotational stress rate $\dot{s}_{i j}^{r}$ and an additional term $N_{i j m n}^{t}\left(\mathrm{I}_{m n k l}-N_{m n k l}^{r}\right) \dot{s}_{k l}$. To clarify the meaning of this additional term, consider again the simple case where the direction of $\sigma_{z}$ is fixed. In this case, $\left(\mathrm{I}_{m n k l}-N_{m n k l}^{r}\right) \dot{s}_{k l}$ represents the part of $\dot{s}_{k l}$ directed along the principal stress directions, i.e. the coaxial part. Recalling the tangential projecting effect of $N_{i j m n}^{t}$, the additional term in $\mathrm{Eq}(18)$ is therefore coaxial as well as tangential to the yield surface in the $\pi$-plane. In other words, it represents a stress rate that is associated with the variation of the Lode angle $\theta$ at fixed $p, \tau$ and principal stress directions. Referring back to Fig 1 , it can be concluded that whilst the RL mechanism is succinctly illustrated in Fig 1(b), the TL mechanism actually includes both conditions illustrated in Figs 1(a) and 1(b).

\section{Numerical simulations}

\subsection{Numerical implementation}

The constitutive models presented above are implemented into ABAQUS finite element software as user-defined material subroutines. The integration of soil models can be conducted by using explicit and implicit schemes, and each of them has its characteristics (Sloan, 1987, Abbo, 1997, Yang et al., 2011, Rezania et al., 2014). For the yield vertex TL model and principal stress RL model, the explicit scheme has been employed throughout to integrate them by the authors, and it proves to be an effective integration scheme (Yang and Yu, 2006, Yang and Yu, 2010, Yang et al., 2011, Yang and Yu, 2013). It is characterized with automatic substepping and error control, leading to robustness and unconditional convergence. For example, it takes as few as two steps to simulate the entire load-displacement 
response by using this explicit scheme (Yang and $\mathrm{Yu}, 2010)$. Therefore, this explicit scheme is employed to integrate these two models in the paper. While reference can be made to Yang and $\mathrm{Yu}$ (2006) and Yang and Yu (2010) for detailed description of the explicit integration, a brief introduction is given below.

For a given strain increment $\Delta \varepsilon_{\mathrm{n}}$ at step $n$, the constitutive equations are firstly integrated over $\Delta \varepsilon_{\mathrm{n}}$ using the first-order Euler scheme to obtain the stress increment $\Delta \sigma_{1}$, followed by using the secondorder modified Euler scheme to obtain the stress increment $\Delta \sigma_{2}$. A local truncation error $R_{\mathrm{n}}$ is determined by the difference between the results of the two schemes. If $R_{\mathrm{n}}$ is larger than a prescribed tolerance STOL, the computation restarts with a smaller increment size $q \Delta \varepsilon_{\mathrm{n}}$ where $q=\max \left[0.9 \sqrt{S T O L / R_{n}}, 0.1\right]$. If $R_{\mathrm{n}}$ is smaller than $S T O L$, the stress state is updated to $\sigma_{n}=\sigma_{n-1}+$ $\left(\Delta \sigma_{1}+\Delta \sigma_{2}\right) / 2$ and the size of the next increment is determined as $\Delta \varepsilon_{\mathrm{n}+1}=q \Delta \varepsilon_{\mathrm{n}}$ where $q=\min \left[0.9 \sqrt{S T O L / R_{n}}, 1.1\right]$. Thus, the algorithm automatically divides the applied strain increment according to the prescribed error tolerance. The foregoing procedure repeats until all strain increments are applied. In addition, the algorithm also considers the yield surface intersection, the occurrence of negative loading index and the yield surface drift correction. Further details can be found in Sloan (1987) and Abbo (1997).

In the following sections, analyses of the simple shear and strip footing problems are carried out to assess the models' performances. The soil used has an elastic modulus $E=10000 \mathrm{kPa}$, a Poisson's ratio $\nu=0.3$ and an initial static lateral earth pressure coefficient $K_{0}=0.5$. The soil cohesion $c$ is set to $1 \mathrm{kPa}$, and the friction angle $\phi$ and dilation angle $\psi$ are both $30^{\circ}$. The associated flow rule is selected in order to avoid possible numerical problems in the strip footing analysis.

\subsection{Simple shear}

A soil element undergoing drained simple shear deformation is considered. A constant vertical stress $\sigma_{y}=100 \mathrm{kPa}$ is applied throughout the shearing. Fig 2 presents the normalized shear stress-shear strain responses using the TL and RL models with different values of $h / G$. The prediction by the DruckerPrager model is also shown for reference. Fig 3 presents a comparison of the magnitudes of the tangential and rotational plastic strain rates $\dot{\varepsilon}_{i j}^{p t}$ and $\dot{\varepsilon}_{i j}^{p r}$, normalized by the magnitude of the conventional plastic strain rate $\dot{\varepsilon}_{i j}^{p c}$. These figures indicate that, compared with the Drucker-Prager model, the inclusion of either the TL or RL mechanism softens the soil responses. Nevertheless, the difference between the TL and RL model predictions is almost negligible. With a smaller $h / G$, this difference becomes larger, and it can be seen that the TL model produces slightly more plastic strain and a softer stress-strain relation than the RL model.

To give a better quantitative comparison, the variations of the Lode angle $\theta$ and the angle $2 \alpha_{\sigma}$ during simple shearing are shown in Fig 4. For the convenience of comparison, $\theta$ is defined to range between $0^{\circ}$ and $60^{\circ}$ with $0^{\circ}$ standing for triaxial compression and $60^{\circ}$ triaxial extension. The reason for comparing $\theta$ with $2 \alpha_{\sigma}$ instead of with $\alpha_{\sigma}$ is because the principal stress rotation is signalled by varying $2 \alpha_{\sigma}$, not $\alpha_{\sigma}$, over the range of $360^{\circ}$ (see Fig $1(\mathrm{~b})$ ). The impact of RL is triggered by the variation of $2 \alpha_{\sigma}$, whilst the impact of TL is triggered by the variations of both $2 \alpha_{\sigma}$ and $\theta$. It is shown in Fig 4 that, in the simple shear, the rotation of principal stress directions is predominant over the variation of the Lode angle, making the difference between $\dot{s}_{i j}^{t}$ and $\dot{s}_{i j}^{r}$ very small. In addition, it is interesting to note that the curve of $2 \alpha_{\sigma}$ predicted by the RL model coincides with that by the TL model, however, the curve of $\theta$ predicted by the RL model coincides with that by the Drucker-Prager model. This discrepancy is another indication to the different loading patterns in the RL and TL mechanisms.

In Fig 5, the deviation of the direction of major principal plastic strain rate $\alpha_{\dot{\varepsilon}} p$ from that of the stress $\alpha_{\sigma}$ is shown to assess the models' capability in reproducing non-coaxiality. Given the fact that slightly more plastic strain is induced by the tangential stress rate $\dot{s}_{i j}^{t}$, it is less non-coaxial than the rotational stress rate $\dot{s}_{i j}^{r}$ owing to the coaxial term in $\dot{s}_{i j}^{t}$, as discussed after $\operatorname{Eq}(18)$. Nevertheless, this difference is again almost negligible in the simple shear.

\subsection{Strip footing}


To further demonstrate the difference between the TL and RL theories, a rigid, rough and strip footing resting on weightless soil is analysed in this section. The finite element mesh is shown in Fig 6. A surcharge load of $\mathrm{q}=100 \mathrm{kPa}$ is applied on the top of the domain, and a prescribed vertical downward displacement is applied to the nodes immediately underneath the footing.

Fig 7 presents the computed footing load-displacement curves using the Drucker-Prager, TL and RL models. Two values of $h / G=0.5$ and 0.2 are used. These two values of $h$ have already been used in the simple shear analysis (see Fig 2), but the results are almost identical between the TL and RL model predictions. However, in the strip footing problem, a significant difference is observed, with the TL model predictions being considerably softer than those by the RL model. This is obviously due to the different loading patterns in the simple shear and strip footing. The former is a simple monotonic type of loading predominated by the change of shear stress, whereas the latter is a complicated case with different locations exposed to different and varying loading patterns consisting of changes in both normal and shear stresses.

To demonstrate this, Figs 8 and 9 show the normalized vertical stress and the variation of $\theta$ and $2 \alpha_{\sigma}$ at four different locations marked in the enlarged diagram of Fig 6. It is seen that the variation of $2 \alpha_{\sigma}$ (contributes to the RL as well as TL) is as small as $7^{\circ}$ at location 1 and around $20^{\circ}$ at location 3 , but the variation of $\theta$ (contributes only to the TL) at these locations is as large as $46^{\circ}$ and therefore is predominant over $2 \alpha_{\sigma}$. As a result, the TL predictions are considerably softer than that by the at these two locations. On the other hand, at location 2 , the $2 \alpha_{\sigma}$ is rapidly increased to more than $65^{\circ}$, whereas the variation of $\theta$ is maintained at around $46^{\circ}$. Consequently, the difference between the TL and RL predictions is smaller at location 2, if compared with that at location 1 . This is also seen for location 4 compared with location 3 . These results are consistent with the different loading patterns associated with the TL and RL mechanisms. Apparently, in shear dominated conditions, the TL and RL theories are almost identical and interchangeable. However, in non-shear dominated conditions where the normal stress change plays an important role as much as the shear stress change, they are not.

With the foregoing comparisons being presented, it can be concluded that, since the TL theory includes the RL, it is more versatile in applications. However, the respective contributions from the RL and the Lode angle variation are not clear. If the research focus is the pure principal stress rotation, the RL theory serves the purpose better since the Lode angle variation effect is eliminated.

\section{Conclusion}

The yield-vertex TL theory is a widely used approach in the constitutive modelling of soil response under principal stress rotational loading. However, the tangential stress rate $\dot{s}_{i j}^{t}$ employed in this theory is not solely designated to represent principal stress rotation. The theory of isolating the rotational stress rate $\dot{s}_{i j}^{r}$ is the true representation of the pure rotation of principal stress axes.

This paper presents a comparative study between the yield-vertex TL and the true principal stress RL theories. Mathematical derivation and numerical simulations are carried out. The following conclusions are made:

1. The tangential stress rate includes the rotational stress rate and an additional coaxial term that is associated with the variation of the Lode angle.

2. The TL theory can be more versatile, but the respective contributions from the RL and the Lode angle variation are not clear in this theory. For the study of pure principal stress rotation, the RL theory serves the purpose better since the Lode angle variation effect is eliminated.

3. Inclusion of either theory can soften the soil stress-strain response and therefore, ignoring them would result in unsafe design in geotechnical problems.

4. In shear dominated problems, such as the simple shear, the two theories give almost identical results since the rotation of principal stress is predominant over the variation of the Lode angle.

5. In non-shear dominated problems, such as the strip footing, the TL theory produces considerably softer results than the RL theory because the normal stress change leading to the variation of the Lode angle has a large impact as well as the principal stress rotation. 


\section{Acknowledgements}

The authors acknowledge the financial support from the International Doctoral Innovation Centre, Ningbo Education Bureau, Ningbo Science and Technology Bureau, and the University of Nottingham. This work was also supported by the UK Engineering and Physical Sciences Research Council (Grant No. EP/G037345/1 and EP/L016362/1], NSFC (Project code 11872219), Zhejiang Natural Science Foundation (project code Q19E090004) and Ningbo Natural Science Programme (project code 2018A610351). These supports are appreciated.

\section{References}

Abbo, A. J. 1997. Finite element algorithms for elastoplasticity and consolidation. PhD. thesis, University of Newcastle Upon Tyne, Australia.

Hashiguchi, K. 2014. Elastoplasticity theory. Springer, Berlin.

Hashiguchi, K. \& Tsutsumi, S. 2001. Elastoplastic constitutive equation with tangential stress rate effect. International Journal of Plasticity, 17, 117-145.

Li, X. \& Dafalias, Y. 2004. A constitutive framework for anisotropic sand including non-proportional loading. Geotechnique, 54, 41-55.

Li, X. \& Yu, H.-S. 2010. Numerical investigation of granular material behaviour under rotational shear. Géotechnique, 60, 381-394.

Miura, K., Miura, S. \& Toki, S. 1986. Deformation behavior of anisotropic dense sand under principal stress axes rotation. Soils and Foundations, 26, 36-52.

Papamichos, E. \& Vardoulakis, I. 1995. Shear band formation in sand according to non-coaxial plasticity model. Geotechnique, 45, 649-661.

Qian, J.-G., Du, Z.-B. \& Yin, Z.-Y. 2017. Cyclic degradation and non-coaxiality of soft clay subjected to pure rotation of principal stress directions. Acta Geotechnica, 1-17.

Qian, J., Yang, J. \& Huang, M. 2008. Three-dimensional noncoaxial plasticity modeling of shear band formation in geomaterials. Journal of engineering mechanics, 134, 322-329.

Rezania, M., Sivasithamparam, N. \& Nezhad, M. M. 2014. On the stress update algorithm of an advanced critical state elasto-plastic model and the effect of yield function equation. Finite Elements in Analysis and Design, 90, 74-83.

Rudnicki, J. W. \& Rice, J. 1975. Conditions for the localization of deformation in pressure-sensitive dilatant materials. Journal of the Mechanics and Physics of Solids, 23, 371-394.

Sloan, S. W. 1987. Substepping schemes for the numerical integration of elastoplastic stress-strain relations. International Journal for Numerical Methods in Engineering, 24, 893-911.

Tian, Y. \& Yao, Y.-P. 2018. Constitutive modeling of principal stress rotation by considering inherent and induced anisotropy of soils. Acta Geotechnica, 1-13.

Tong, Z.-X., Zhang, J.-M., Yu, Y.-L. \& Zhang, G. 2010. Drained deformation behavior of anisotropic sands during cyclic rotation of principal stress axes. Journal of Geotechnical and Geoenvironmental Engineering, 136, 1509-1518.

Tsutsumi, S. \& Hashiguchi, K. 2005. General non-proportional loading behavior of soils. International Journal of Plasticity, 21, 1941-1969.

Yang, Y. \& Yu, H.-S. 2006. Numerical simulations of simple shear with non-coaxial soil models. International journal for numerical and analytical methods in geomechanics, 30, 1-19.

Yang, Y. \& Yu, H.-S. 2010. Numerical aspects of non-coaxial model implementations. Computers and Geotechnics, 37, 93-102.

Yang, Y., Yu, H.-S. \& Kong, L. 2011. Implicit and explicit procedures for the yield vertex non-coaxial theory. Computers and Geotechnics, 38, 751-755.

Yang, Y. \& Yu, H. S. 2013. A kinematic hardening soil model considering the principal stress rotation. International Journal for Numerical and Analytical Methods in Geomechanics, 37, 2106-2134.

Yatomi, C., Yashima, A., Iizuka, A. \& Sano, I. 1989. General theory of shear bands formation by a non-coaxial Cam-clay model. Soils and foundations, 29, 41-53.

Yu, H.-S. 2006. Plasticity and geotechnics. Springer, New York. 
Yu, H. \& Yuan, X. 2006. On a class of non-coaxial plasticity models for granular soils. Proceedings of the Royal Society of London A: Mathematical, Physical and Engineering Sciences. 462, 725748.

Yuan, R., Yu, H.-S., Hu, N. \& He, Y. 2018. Non-coaxial soil model with an anisotropic yield criterion and its application to the analysis of strip footing problems. Computers and Geotechnics, 99, $80-92$. 


\section{FIGURES}
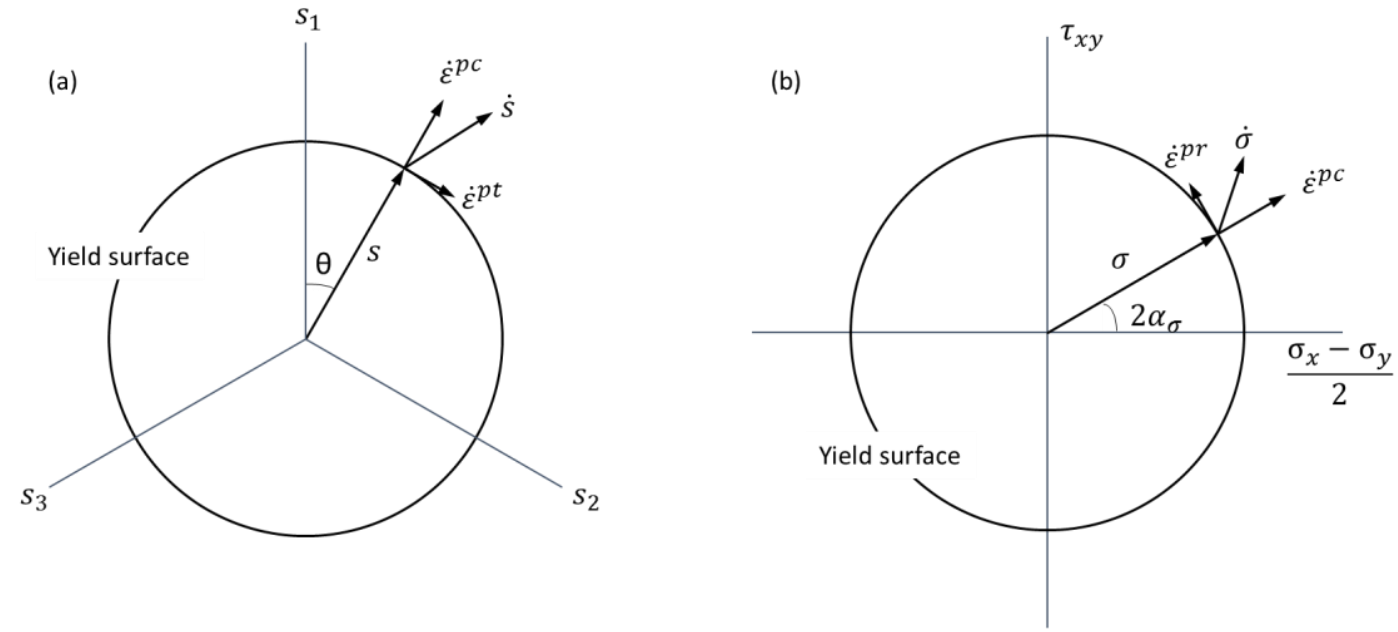

Fig 1. Schematic illustration of the (a) tangential plastic strain rate $\dot{\varepsilon}_{i j}^{p t}$ in the $\pi$-plane and (b) rotational plastic strain rate $\dot{\varepsilon}_{i j}^{p r}$ in the plane of $\left(\frac{\sigma_{x}-\sigma_{y}}{2}, \tau_{x y}\right)$. 


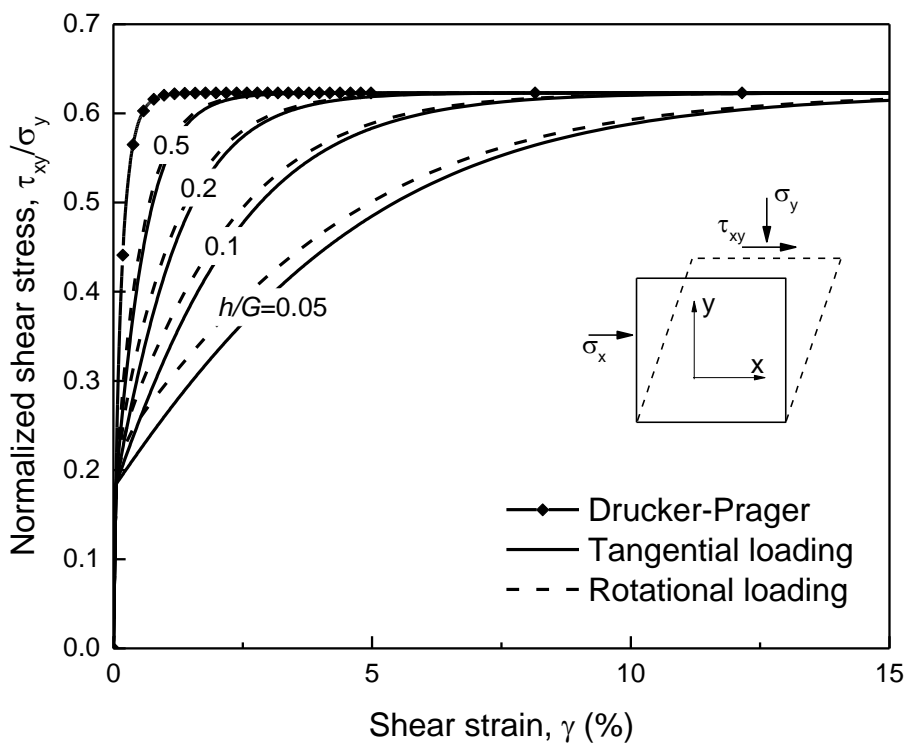

Fig 2. Normalized shear stress-shear strain responses during simple shear. 


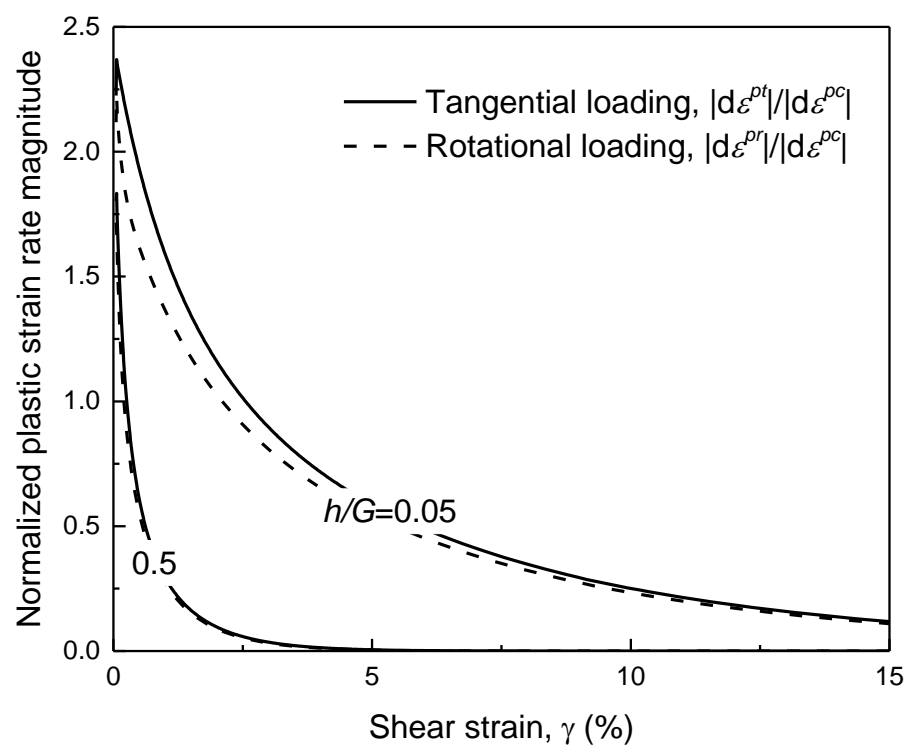

Fig 3. Normalized plastic strain rate magnitudes. 


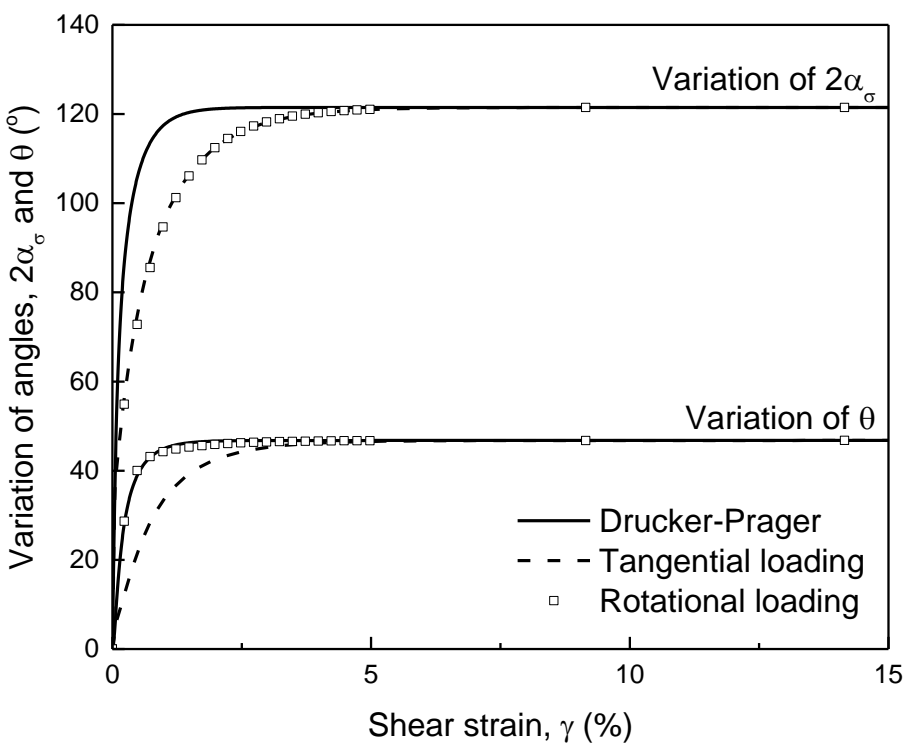

Fig 4. Variations of $2 \alpha_{\sigma}$ and Lode angle $\theta$. 


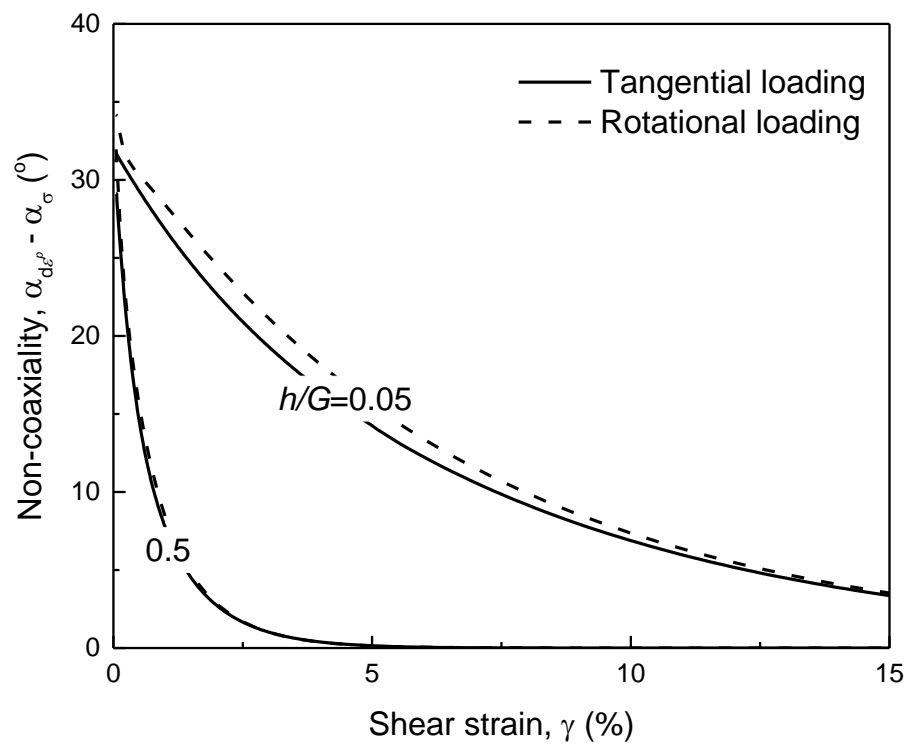

Fig 5. Degree of non-coaxiality, $\alpha_{\dot{\varepsilon}} p-\alpha_{\sigma}$. 


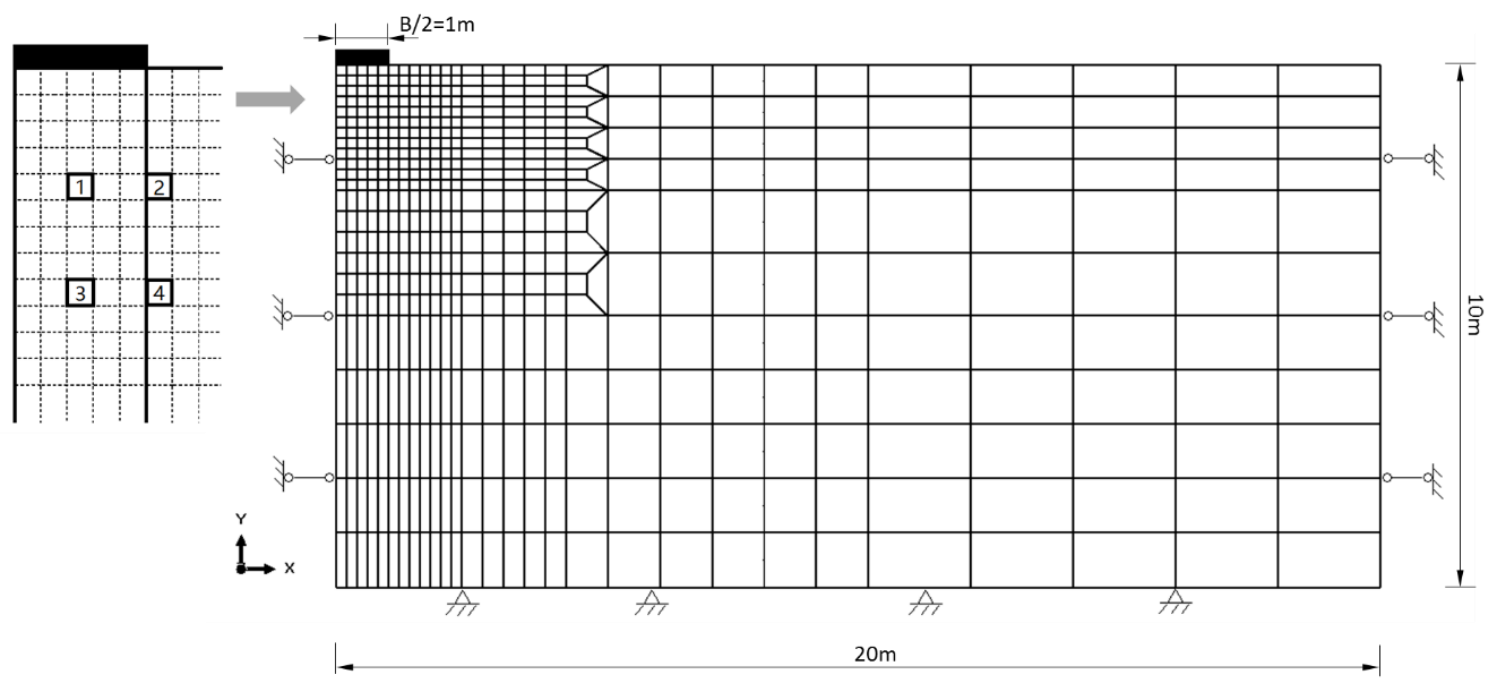

Fig 6. Finite element mesh for strip footing problem. 

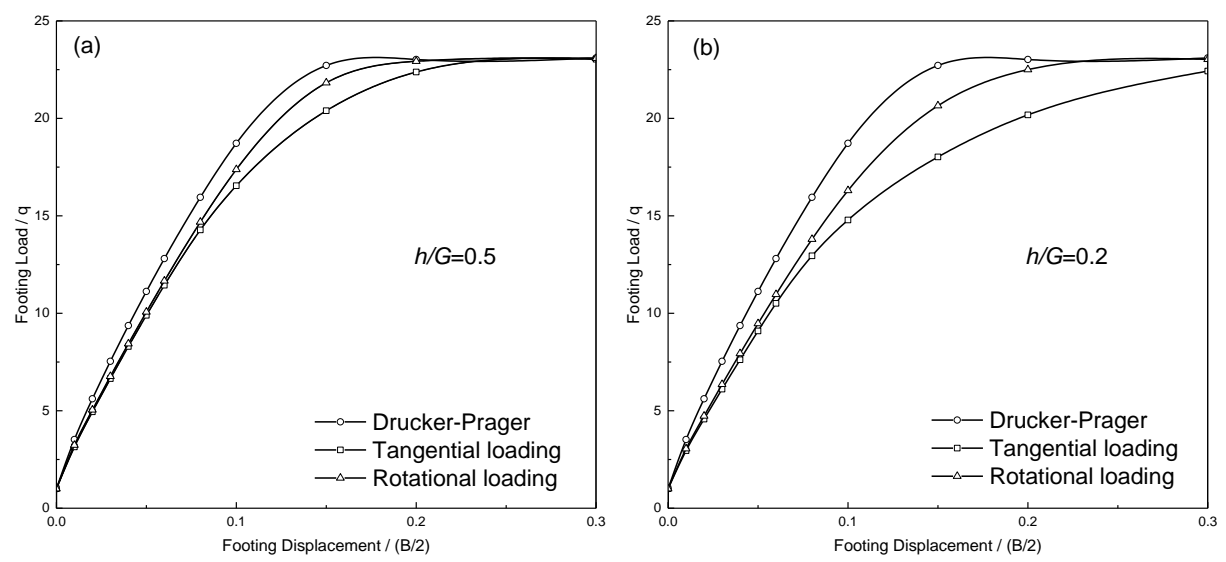

Fig7. Predicted footing load-displacement curves. 

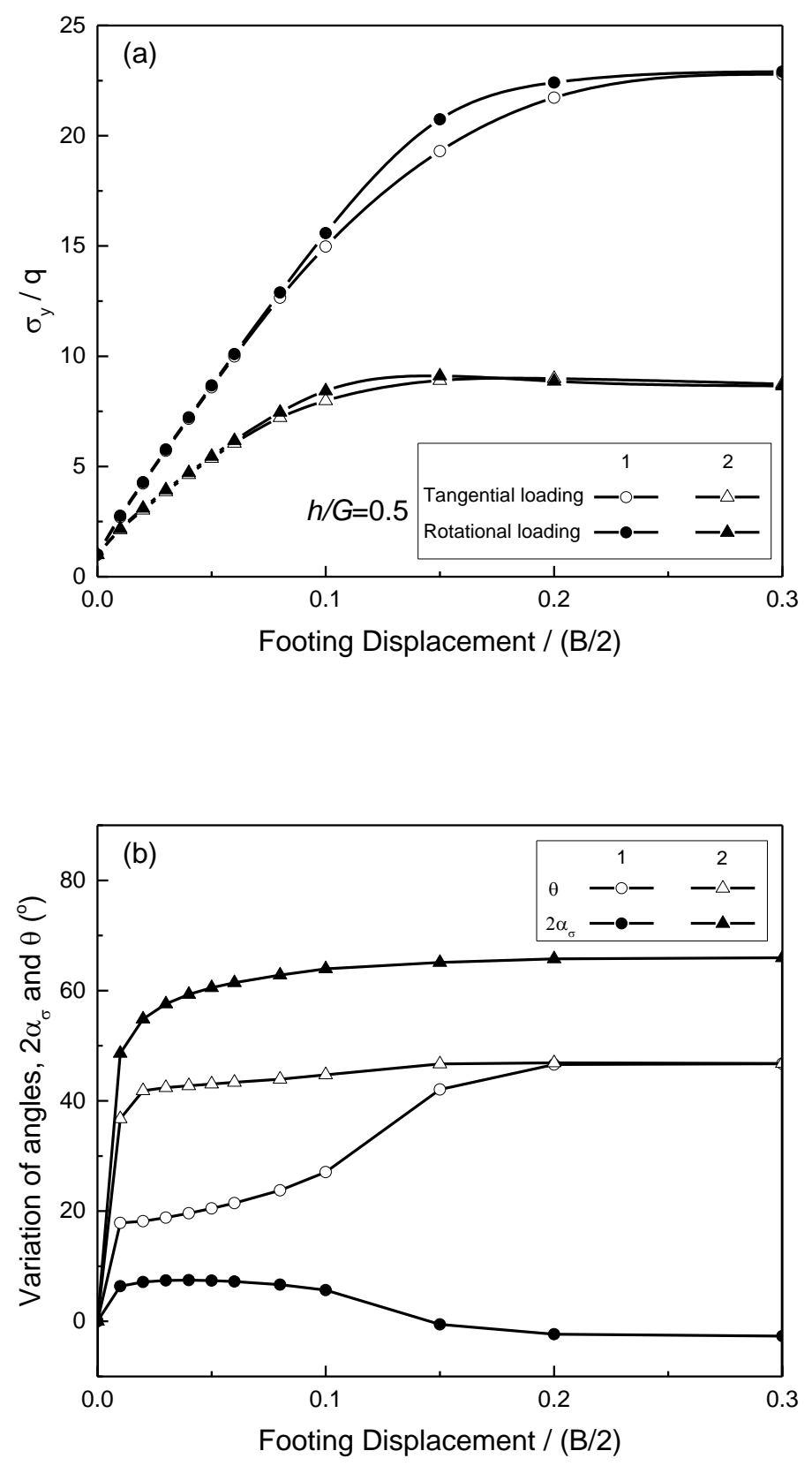

Fig 8. Predicted results at locations 1 and 2: (a) normalized vertical stress predicted by TL and RL models with $h / G=0.5$, and (b) variations of $\theta$ and $2 \alpha_{\sigma}$. 

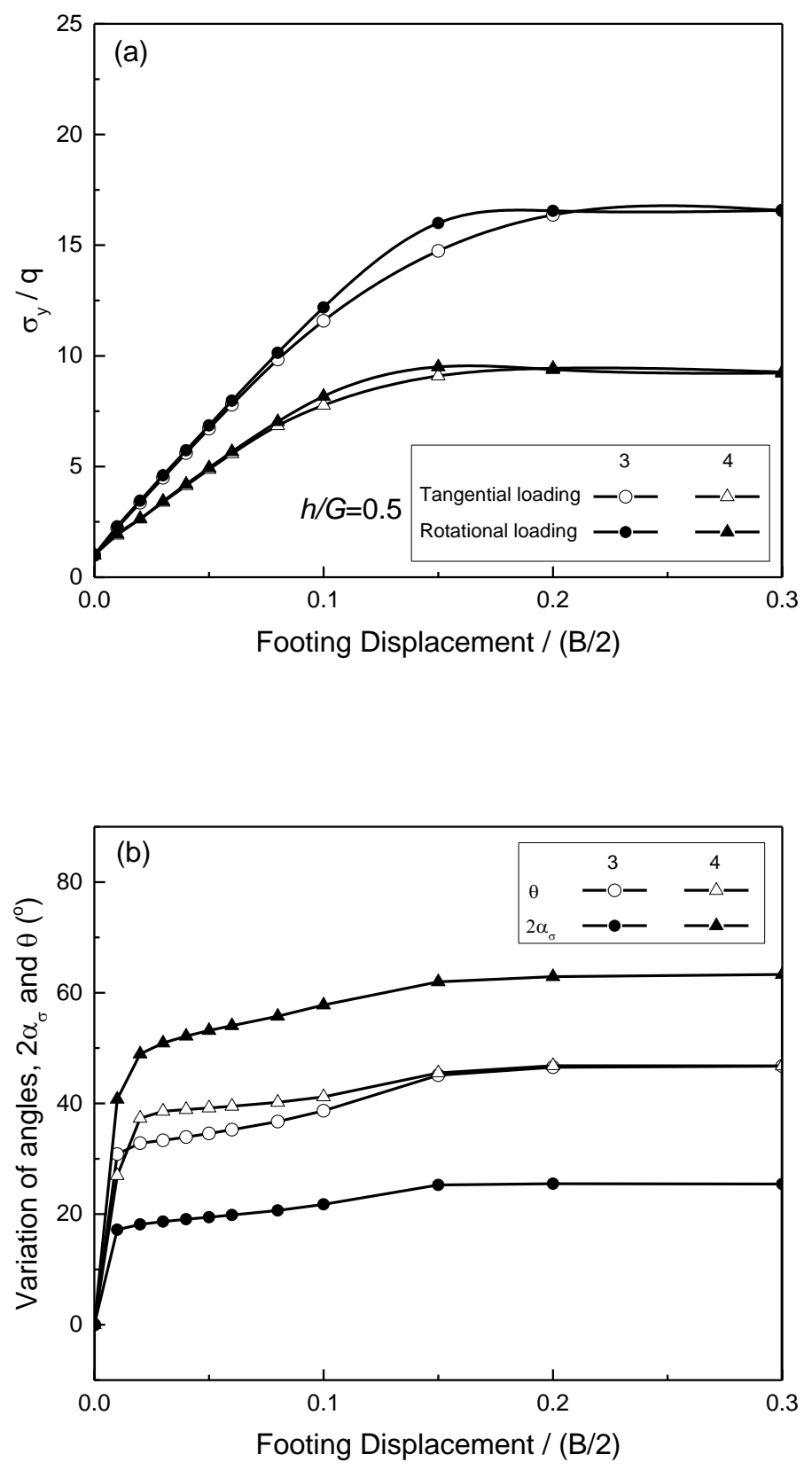

Fig 9. Predicted results at locations 3 and 4: (a) normalized vertical stress predicted by TL and RL models with $h / G=0.5$, and (b) variations of $\theta$ and $2 \alpha_{\sigma}$. 